\title{
Guidelines and Performance Measures for the Management of Acute Coronary Syndrome
}

\author{
Piotr Sobieszczyk, MD
}

\begin{abstract}
BACKGROUND: Acute coronary syndrome (ACS) is caused by reduced perfusion of the myocardium and characterized by chest pain. The primary goals of treatment for ACS are to restore blood flow through occluded coronary arteries and prevent recurrent coronary events. Antiplatelet and anticoagulant therapies play a crucial role in the treatment of ACS by interrupting the thrombotic process.
\end{abstract}

OBJECTIVES: To characterize the pathophysiology of ACS and to describe the American College of Cardiology (ACC)/American Heart Association (AHA) guidelines for reperfusion therapy in patients with ST-segment elevation myocardial infarction (STEMI); the use of antiplatelet and anticoagulant therapies for the treatment of STEMI, non-ST-segment elevation myocardial infarction (NSTEMI), and unstable angina (UA), including patients who undergo percutaneous coronary intervention (PCI); and long-term antiplatelet therapy after an ACS episode.

SUMMARY: The preferred reperfusion strategy in patients with STEMI is $\mathrm{PCl}$ if it can be performed within 90 minutes after arrival at the hospital. Patients with NSTEMI or UA also may undergo PCI. Thrombolysis is an alternative method of reperfusion for patients with STEMI but not for patients with NSTEMI or UA. Dual antiplatelet therapy with aspirin and the thienopyridine clopidogrel is recommended by the ACC/AHA for 12 months in patients with STEMI, NSTEMI, or UA, including patients with coronary stents. Platelet glycoprotein (GP) Ilb/Illa inhibitors are routinely used during $\mathrm{PCI}$ in patients with STEMI and NSTEMI. These agents should be used in addition to aspirin and clopidogrel in patients with NSTEMI and elevated troponin levels who undergo coronary intervention. Unfractionated heparin (UFH) or the low-molecular-weight heparin enoxaparin may be used in patients with STEMI undergoing reperfusion with thrombolytic agents or PCI and patients with NSTEMI or UA. There are substantial data on enoxaparin in the ACS arena, but UFH is preferred for patients who undergo coronary intervention because of greater ease of therapeutic monitoring and reversal of anticoagulant effects if bleeding complications arise. The pentasaccharide fondaparinux may become an alternative to UFH and enoxaparin for patients with STEMI and some patients with NSTEMI or UA. Fondaparinux is preferred for patients with NSTEMI or UA who are at increased risk for bleeding when a conservative approach is chosen, but it is not recommended for patients when an early invasive approach is chosen because of the risk of catheter-related thrombi. The direct thrombin inhibitor bivalirudin may be used for anticoagulation in patients with NSTEMI who undergo early invasive procedures, and GP IIb/IIla inhibitor use may be avoided in some patients; however, upstream antiplatelet therapy with clopidogrel is also needed if these patients undergo PCI. Patients undergoing coronary stenting must receive dual antiplatelet therapy.

CONCLUSIONS: Antiplatelet and anticoagulant therapies for patients with ACS are complex. Evidence-based guidelines facilitate the therapeutic decision-making process for these therapies in patients with ACS.

J Manag Care Pharm. 2008;14(6)(suppl S-a):S4-S13

Copyright@ 2008, Academy of Managed Care Pharmacy. All rights reserved.

\section{Pathophysiology and Epidemiology}

Acute coronary syndrome (ACS) is a manifestation of coronary heart disease (CHD) that encompasses a spectrum of events in which blood flow to the myocardium is suddenly and severely reduced or completely interrupted: ST-segment elevation myocardial infarction (STEMI), non-ST-segment elevation myocardial infarction (NSTEMI), and unstable angina (UA). The decrease in myocardial perfusion causes myocardial ischemia that can lead to cell death and myocardial infarction (MI), which may then result in heart failure and death. The classic symptom of ACS is chest pain. Myocardial injury and cell death can be detected by characteristic changes in the electrocardiogram (ECG) and by elevation of the cardiac proteins troponin and creatine kinase in the bloodstream.

The most dangerous of the 3 clinical scenarios is STEMI, which is often associated with sudden death. It accounts for a little more than one third of ACS cases and is caused by the sudden rupture of a cholesterol-filled plaque in the coronary artery wall. ${ }^{1}$ Exposure of contents of the plaque to constituents in the bloodstream initiates a cascade of events that culminates in the formation of a thrombus over the plaque. The thrombus completely blocks blood flow through the artery, depriving the myocardium of blood and causing infarction. This complete cessation of blood flow to the myocardium causes characteristic elevations in the ST segments on the ECG and can also result in pathological Q waves.

A similar pathophysiological process occurs in patients with NSTEMI, but blockage of the artery, interruption of blood flow by the thrombus, and myocardial cell injury and death are less extensive than in patients with STEMI. Nevertheless, blood flow to the myocardium can be sufficiently reduced to cause ECG changes and the release of troponin and creatine kinase from injured muscle cells. Similar pathology occurs with UA, yet UA is characterized by chest pain at rest lasting for more than 20 minutes or chest pains that rapidly accelerate in frequency and

\section{Author}

PIOTR SOBIESZCZYK, MD, is Associate Director, Cardiac Catheterization Laboratory, Brigham and Women's Hospital, Boston.

AUTHOR CORRESPONDENCE: Piotr Sobieszczyk, MD, Associate Director, Cardiac Catheterization Laboratory, Brigham and Women's Hospital, Cardiovascular Division, PBB-113, 75 Francis St. Boston, MA 02115. Tel.: 617.732.8898; Fax: 617.732.7122 E-mail: psobieszczyk@partners.org 
severity. UA is a warning sign of rapidly diminishing blood flow, which may continue to worsen and progress to MI.

\section{Treatment}

The primary goals of treatment for ACS are to restore blood flow through occluded coronary arteries and prevent recurrent coronary events. Treatment of ACS has evolved over the last several decades. Percutaneous coronary intervention (PCI) is now an integral part of ACS management. The vast majority of patients with ACS undergo PCI with balloon angioplasty to reopen occluded coronary arteries by insertion of a bare-metal or drugeluting stent in the reopened artery to maintain vessel patency. The urgency of $\mathrm{PCI}$ and coronary stenting is dictated by the type of ACS and the clinical condition of the patient.

Medical management of ACS involves antiplatelet and anticoagulant therapies to interrupt the thrombotic process. Reductions in myocardial oxygen demand and potentially lethal arrhythmias are achieved with administration of beta-blockers. Angiotensin-converting enzyme (ACE) inhibitors are used to inhibit ventricular remodeling in the damaged myocardium and prevent the development of heart failure. Nitrates remain the mainstay of therapy for the relief of angina and improvement in myocardial blood flow. Lipid-lowering agents reduce cholesterol levels, decrease plaque formation, and prevent the rupture of existing plaques. Antiplatelet and anticoagulant therapies are the focus of this article.

\section{Reperfusion Therapy in Patients with STEMI}

In patients with STEMI, time is of the essence because rapid reperfusion reduces damage to the myocardium and the risk of heart failure and death. ${ }^{2}$ Salvage of myocardial tissue depends on the early recognition of symptoms and rapid initiation of therapy.

The 2 strategies commonly used for opening an occluded coronary artery are thrombolysis and PCI with coronary stent deployment. PCI is the preferred reperfusion strategy if cardiac catheterization can be performed within 90 minutes after presentation (Figure 1). ${ }^{2}$ Nevertheless, many patients with STEMI are treated in hospitals without cardiac catheterization facilities, and thrombolysis remains an important method of reperfusion in the United States and worldwide. The goal is to administer thrombolytic therapy early after symptom onset and within 30 minutes after the patient presents to the health care system. ${ }^{2}$ However, thrombolytic agents remain effective when used up to 12 hours after symptom onset. ${ }^{3}$

\section{Ancillary Antiplatelet and Anticoagulation Therapies in Patients with STEMI}

After a thrombus is dissolved by thrombolytic therapy and myocardial reperfusion is achieved, plaque contents remain exposed to the bloodstream. This exposure continues to drive the coagulation cascade and induce thrombin deposition, which promotes platelet aggregation on the plaque, recurrence of thrombus formation, and reocclusion of the coronary artery. Therefore, ancillary antiplatelet and anticoagulation therapies should be administered concurrently with thrombolytic agents.

\section{Antiplatelet Therapies}

Antiplatelet therapy has been the cornerstone of ACS management. Aspirin $160 \mathrm{mg}-325 \mathrm{mg}$ per day is recommended initially for patients with STEMI. ${ }^{2,4}$ In a placebo-controlled study of patients with suspected STEMI, enteric-coated aspirin $160 \mathrm{mg}$ per day reduced the 5 -week cardiovascular mortality rate by $23 \%$ compared with placebo. ${ }^{5}$ A nonenteric-coated formulation is recommended for acute events to allow rapid buccal absorption. ${ }^{4}$ Aspirin irreversibly inhibits platelet aggregation by inhibiting the enzyme cyclooxygenase-1, thereby reducing the production of thromboxane $\mathrm{A}_{2}$, one of several potent platelet activators. ${ }^{4}$ Aspirin $75 \mathrm{mg}-325 \mathrm{mg}$ per day should be continued indefinitely in patients with STEMI unless it is contraindicated. ${ }^{2}$ Patients who cannot take aspirin because of an allergy may receive a thienopyridine (e.g., clopidogrel, ticlopidine) instead.

Thienopyridines inhibit platelet aggregation by irreversibly binding to platelet adenosine diphosphate receptors. ${ }^{6}$ Loading doses are needed to provide a prompt antiplatelet effect on the first day of therapy. ${ }^{6}$ Although ticlopidine has been used successfully in patients with STEMI and UA/NSTEMI to prevent coronary stent thrombosis, its use is limited by a high incidence of gastrointestinal (GI) adverse effects and neutropenia. ${ }^{7}$

Clopidogrel does not have the side-effect profile associated with ticlopidine. ${ }^{6}$ Clopidogrel is administered orally as a 300-mg loading dose followed by a 75-mg-per-day maintenance dose. There is new evidence that a larger clopidogrel loading dose of $600 \mathrm{mg}-900 \mathrm{mg}$ can be used safely to accelerate full platelet inhibition. ${ }^{8,9}$

Clopidogrel has been widely used in combination with aspirin after placement of coronary stents or as an alternative to aspirin in patients with ACS. Recent evidence from 2 large randomized, placebo-controlled studies suggests that the combination of aspirin and clopidogrel (dual antiplatelet therapy) is beneficial in patients with STEMI. ${ }^{10,11}$ In the COMMIT trial, $>45,000$ patients with STEMI were randomly assigned to receive clopidogrel $75 \mathrm{mg}$ per day or placebo for up to 4 weeks or until discharge from the hospital. ${ }^{10}$ All patients received aspirin $162 \mathrm{mg}$ per day. The incidence of death, reinfarction, or stroke was significantly lower in the clopidogrel group (9.2\%) compared with the placebo group (10.1\%, $P=0.002$ ). All-cause mortality was significantly lower in the clopidogrel group $(7.5 \%)$ than in the placebo group $(8.1 \%$, $P=0.030$ ). The benefit of clopidogrel was independent of thrombolytic therapy, which was used in 54\% of enrollees. No excess risk of cerebral or fatal bleeding was associated with clopidogrel.

In the CLARITY-TIMI 28 trial, 3,491 patients who received thrombolytic therapy within 12 hours after STEMI onset were randomly assigned to receive clopidogrel $300 \mathrm{mg}$ followed by 


\section{FIGURE Use of Antiplatelet and Anticoagulant Therapies in Patients with ST-Segment Elevation} Myocardial Infarction

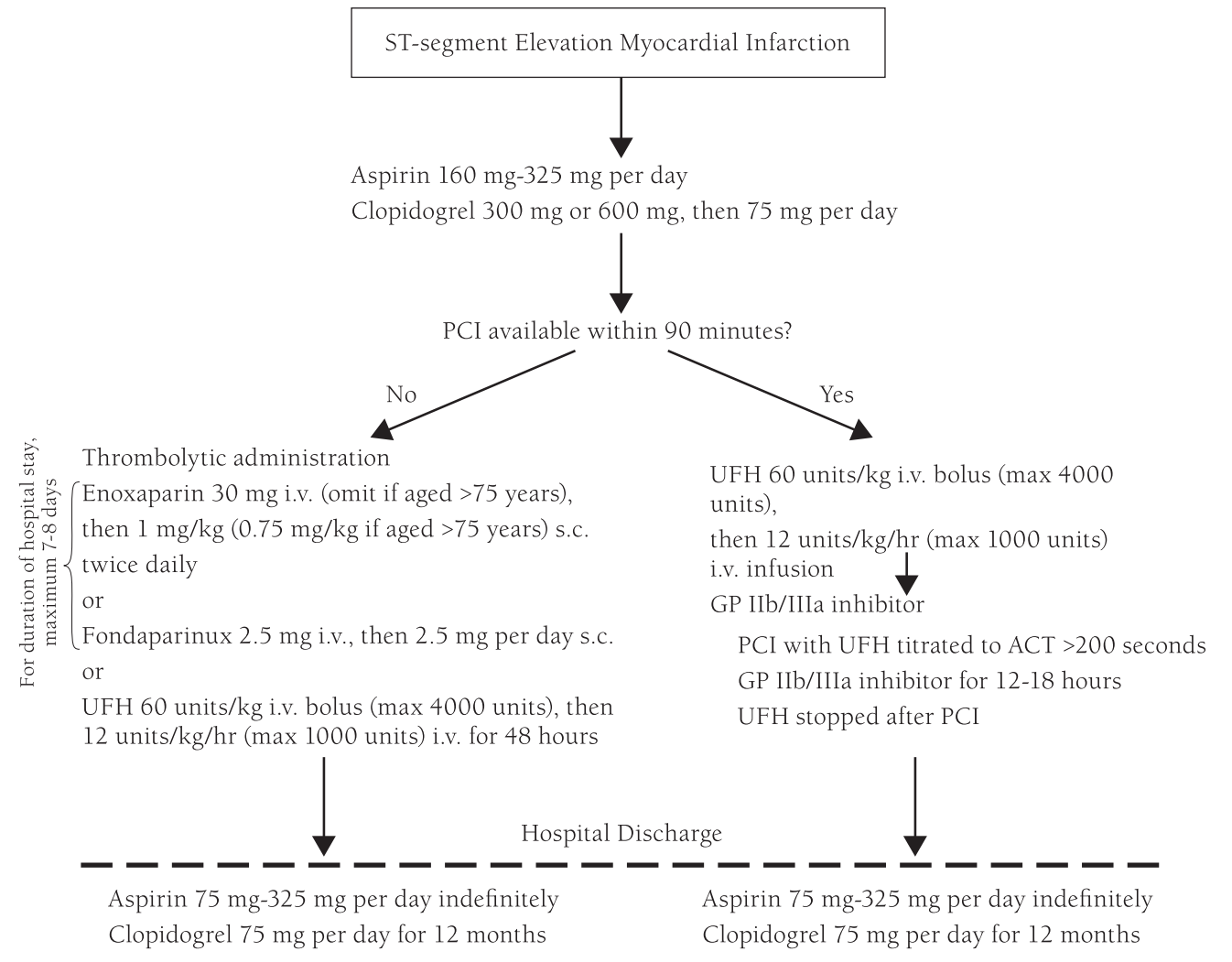

Adapted from Antman et al. ${ }^{2}$

$A C T=$ activated clotting time; $G P=$ glycoprotein; i.v.=intravenous; $P C I=$ percutaneous coronary intervention; s.c. =subcutaneous; $U F H=u n f r a c t i o n a t e d ~ h e p a r i n$.

$75 \mathrm{mg}$ per day or placebo. ${ }^{11}$ All patients received aspirin $150 \mathrm{mg}-325 \mathrm{mg}$ on the first day followed by $75 \mathrm{mg}-162 \mathrm{mg}$ per day. The rate of recurrent MI, death, or persistently occluded artery during subsequent coronary angiography was significantly lower in the clopidogrel group $(15.0 \%)$ than in the placebo group $(21.7 \%, P<0.001)$. Clopidogrel primarily affected the rate of recurrent MI and persistent artery occlusion, not all-cause mortality. The rate of major bleeding after 30 days was similar in the clopidogrel group (1.9\%) and the placebo group (1.7\%, $P=0.800$ ).

Results of the CLARITY-TIMI 28 and COMMIT trials suggest that clopidogrel should be administered in combination with aspirin in patients with STEMI regardless of whether thrombolytic agents are used. Current evidence-based guidelines of the American College of Cardiology (ACC) and American Heart
Association (AHA) support this practice, although no data are available to guide decision making about a loading dose of clopidogrel for patients aged $\geq 75$ years. ${ }^{2}$ The optimal duration of clopidogrel therapy in patients with STEMI is unclear. ${ }^{2}$ Current ACC/ AHA recommendations support a 12-month course of dual antiplatelet therapy. ${ }^{2}$ The increased risk of bleeding associated with dual antiplatelet therapy is a concern in patients who are planning to undergo coronary artery bypass graft (CABG) surgery. Clopidogrel should be withheld for at least 5 days and preferably 7 days before CABG surgery to reduce the risk of operative bleeding. ${ }^{2}$

\section{Anticoagulant Therapies}

Patients undergoing thrombolysis should receive ancillary anticoagulation therapy to reduce the risk of reinfarction. 
Unfractionated heparin (UFH) therapy should be administered for a minimum of 48 hours, and enoxaparin and fondaparinux should be used for the duration of hospitalization up to 8 days. ${ }^{2}$ The traditional anticoagulant is UFH, a mixture of polysaccharide chains that promote the activity of circulating antithrombin. Antithrombin, in turn, inactivates thrombin (factor IIa) and factor Xa. ${ }^{12}$ UFH prevents thrombus propagation but does not dissolve an existing thrombus. UFH is administered during thrombolysis as an intravenous bolus dose of 60 units per $\mathrm{kg}$ (not to exceed 4,000 units) followed by intravenous infusion of 12 units per kg per hour (not to exceed 1,000 units per hour). ${ }^{2}$ The dosage is titrated to maintain an activated partial thromboplastin time (aPTT) 1.5-2.0 times that of a specified control value (50-70 seconds). ${ }^{2}$ Anticoagulants other than UFH are recommended if therapy is continued for more than 48 hours. $^{2}$ Continuing UFH therapy for more than 48 hours increases the risk of heparin-induced thrombocytopenia (HIT), an immunemediated reduction in platelet count that increases the risk for thrombosis. ${ }^{2}$

The need for continuous intravenous infusion, frequent laboratory monitoring, and dosage adjustment, as well as difficulty achieving consistent levels of anticoagulation stimulated a search for anticoagulants that are as effective and safe as UFH but easier to use. Chemical or enzymatic modification of UFH yielded low-molecular-weight heparin (LWMH) with smaller polysaccharide chains. LMWH inactivates factor Xa to a greater extent than factor IIa, and it does not prolong the aPTT. ${ }^{12}$ The risk for HIT is lower in patients receiving LMWH than in patients treated with UFH. ${ }^{12}$ LMWH binds to plasma proteins to a lesser extent than UFH, which improves the predictability of the dose-response relationship. ${ }^{12}$ LMWH also has a longer duration of pharmacodynamic effects than UFH. Most LMWH products are administered once or twice daily without need for laboratory monitoring.

The most extensively studied LMWH in patients with STEMI is enoxaparin. In patients aged $<75$ years and without severe renal impairment, enoxaparin is administered at the time of thrombolysis as a 30-mg intravenous dose followed 15 minutes later by subcutaneous injection of $1 \mathrm{mg}$ per kg every 12 hours for the duration of hospitalization up to 8 days. ${ }^{2}$ Patients aged $\geq 75$ years may be at increased risk for bleeding during enoxaparin therapy; therefore, the initial intravenous dose should be omitted, and a reduced maintenance dosage of $0.75 \mathrm{mg}$ per $\mathrm{kg}$ subcutaneously every 12 hours should be used., ${ }^{2,13}$ A maintenance dosage of $1 \mathrm{mg}$ per $\mathrm{kg}$ subcutaneously every 24 hours should be used if the estimated creatinine clearance is $<30 \mathrm{~mL}$ per minute regardless of age. ${ }^{2}$

Enoxaparin was compared with UFH in a randomized, openlabel study of 4,078 patients with STEMI known as ASSENT-3. ${ }^{13}$ Patients received tenecteplase plus enoxaparin $30 \mathrm{mg}$ intravenously followed immediately by $1 \mathrm{mg}$ per $\mathrm{kg}$ subcutaneously every 12 hours until hospital discharge or revascularization for up to 7 days, or they received tenecteplase plus UFH 60 units per $\mathrm{kg}$ (not to exceed 4,000 units) as an intravenous bolus followed by 12 units per kg per hour (not to exceed 1,000 units per hour) by intravenous infusion with the dosage adjusted to maintain an aPTT of 50-70 seconds for 48 hours. The incidence of a composite endpoint of 30-day mortality, in-hospital reinfarction, or in-hospital refractory ischemia was significantly lower in the enoxaparin group (11.4\%) than in the UFH group (15.4\%, $P=0.001)$. Similar findings were observed when this composite endpoint included in-hospital intracranial bleeding and in-hospital major bleeding complications (13.7\% with enoxaparin vs. $17.0 \%$ with UFH, $P=0.008$ ). However, patients aged $\geq 75$ years who received enoxaparin were at a slightly higher risk for bleeding that was not significant compared with patients of the same age who received UFH. These findings form part of the basis for the ACC/AHA guidelines for enoxaparin dosage reduction to prevent bleeding complications in elderly patients. ${ }^{2}$

In a more recent randomized, double-blind study known as EXTRACT-TIMI 25, enoxaparin was compared with UFH in more than 20,000 patients with STEMI who were planning to undergo thrombolysis. ${ }^{14}$ The ACC/AHA dosing recommendations for enoxaparin in patients aged $\geq 75$ years and younger patients and UFH were followed. The 30-day rate of death or nonfatal recurrent MI was $9.9 \%$ in the enoxaparin group and $12 \%$ in the UFH group, a difference that is significant $(P<0.001)$. The incidence of major bleeding after 30 days was significantly higher in the enoxaparin group (2.1\%) than in the UFH group $(1.4 \%, P<0.001)$. When results were stratified by age, the relative risk of major bleeding in patients aged $\geq 75$ years treated with enoxaparin instead of UFH (1.15) was lower than that found in younger patients (1.67). ${ }^{15}$

Fondaparinux is a synthetic pentasaccharide that inhibits factor Xa activity, thrombin generation, and thrombus formation by selectively binding to antithrombin III. ${ }^{12}$ The drug has a long half-life that allows once-daily administration. In patients with STEMI, fondaparinux is given as an initial 2.5-mg intravenous dose followed by $2.5 \mathrm{mg}$ subcutaneously once daily. ${ }^{2}$ This usage is not approved by the U.S. Food and Drug Administration (FDA) ${ }^{16}$ The level of anticoagulation provided by fondaparinux is consistent, and laboratory monitoring is not required. Fondaparinux is contraindicated in patients with severe renal impairment (creatinine clearance $<30 \mathrm{~mL}$ per min) because the drug is eliminated primarily by the kidneys, and patients with severe renal impairment are at increased risk for major bleeding. ${ }^{16}$ Currently, no antidote is available to reverse the anticoagulant effects of fondaparinux; therefore, the drug's long half-life may be detrimental when bleeding occurs.

Fondaparinux $2.5 \mathrm{mg}$ intravenously followed by $2.5 \mathrm{mg}$ subcutaneously once daily for up to 8 days was compared with UFH given in accordance with ACC/AHA guidelines for up to 48 hours or with placebo in the OASIS- 6 trial, a randomized, double-blind study of more than 12,000 patients with STEMI. ${ }^{17}$ 
Thrombolytic therapy was used in $45 \%$ of patients in both treatment groups. The 30-day incidence of death or reinfarction was significantly lower in the fondaparinux group (9.7\%) than in the control group (11.2\%, $P=0.008)$. The incidence of severe hemorrhage was slightly lower in the fondaparinux group (1.0\%) than in the control group (1.3\%), but the difference was not significant $(P=0.13)$.

Fondaparinux has not been compared with LMWH in well-controlled clinical trials of patients with STEMI, nor has its safety in combination with dual antiplatelet therapy been evaluated. At present, the ACC/AHA guidelines provide the strongest support for the use of UFH, enoxaparin, or fondaparinux in patients with STEMI undergoing reperfusion with thrombolytic agents. ${ }^{2}$

\section{- Treatment Strategies for NSTEMI/UA}

The basic principles of pharmacotherapy for patients with NSTEMI or UA are similar to those for patients with STEMI. ${ }^{18}$ Use of beta-blockers, ACE inhibitors, nitrates, and aspirin in patients with NSTEMI or UA is as important as it is in patients with STEMI. The primary difference in treatment regimens for these patient populations lies in the reperfusion strategies.

In patients with NSTEMI or UA, thrombolytic agents are uniformly harmful. The partly blocked coronary artery is treated with a combination of antiplatelet agents and anticoagulants. The goal of this approach is to stabilize the ruptured plaque and prevent complete occlusion of the coronary vessel by interrupting the coagulation cascade and preventing thrombus progression. The intensity of anticoagulation can be increased if the clinical status worsens.

In the United States, most patients with NSTEMI or UA undergo cardiac catheterization within the first 24-48 hours after presentation; therefore, the compatibility of the anticoagulants used during this initial period with the needs of the cardiac catheterization laboratory is a consideration. The desired characteristics of anticoagulants used in the cardiac catheterization laboratory differ from those used in the medical management of NSTEMI or UA.

A system for predicting the risk for death and ischemic events in patients with NSTEMI or UA known as the Thrombolysis in Myocardial Infarction (TIMI) risk scoring system has been developed based on clinical trial data in this patient population. ${ }^{19}$ This risk scoring system is one of several systems for risk stratification that are used for planning therapeutic strategies in patients with NSTEMI or UA based on risk. In the TIMI risk scoring system, 1 point is given to each of 7 variables: aged $\geq 65$ years, at least 3 CHD risk factors, prior coronary stenosis $\geq 50 \%$, ST-segment deviation on the ECG at time of presentation, at least 2 anginal events in the preceding 24 hours, use of aspirin in the preceding 7 days, and elevated serum cardiac markers. A patient's TIMI risk is categorized based on the number of points as low (0-2), medium (3-4), or high (5-7). Patients with
NSTEMI or UA and a low TIMI risk score are usually evaluated in the emergency department using serial biochemical marker measurements. Patients with negative test results typically are admitted to a general floor of the hospital with telemetric monitoring, and they may undergo stress testing to ascertain the need for cardiac catheterization. Most patients with NSTEMI or UA and a medium or high TIMI risk score are admitted to a coronary intensive care unit, chest pain unit, or general floor depending on the perceived risk. High-risk patients undergo early PCI and revascularization if stenosis is found. Most moderate-risk patients also undergo PCI and revascularization during the hospital stay, although patients without MI may have a stress test before PCI.

\section{Antiplatelet Therapies}

Guidelines for the management of NSTEMI and UA that address antiplatelet and anticoagulant therapies have been published by the ACC and AHA. ${ }^{18}$ Three types of antiplatelet agents are used in NSTEMI or UA: aspirin, thienopyridines, and platelet glycoprotein (GP) IIb/IIIa inhibitors.

Aspirin should be administered as soon as possible in the course of medical evaluation of patients with NSTEMI or UA regardless of whether they plan to undergo cardiac catheterization. $^{18}$ The recommended initial dosage is $162 \mathrm{mg}-325 \mathrm{mg}$ per day in an oral or chewable nonenteric formulation. ${ }^{18}$ Aspirin $75 \mathrm{mg}-325 \mathrm{mg}$ per day should be continued indefinitely in patients without aspirin intolerance or hypersensitivity. ${ }^{18}$ Patients with aspirin intolerance or hypersensitivity should receive clopidogrel $300 \mathrm{mg}-600 \mathrm{mg}$ as a loading dose followed by $75 \mathrm{mg}$ per day instead of aspirin. ${ }^{18}$ Patients with a history of GI bleeding may benefit from addition of a proton pump inhibitor to reduce the risk of bleeding during treatment with aspirin or clopidogrel. $^{18}$

In a randomized, double-blind, placebo-controlled study of more than 12,000 patients with NSTEMI or UA known as the CURE trial, a 300-mg loading dose of clopidogrel followed by $75 \mathrm{mg}$ per day for 3-12 months was associated with a significant reduction in the incidence of death from cardiovascular causes, nonfatal MI, or stroke $(9.3 \%$ vs. $11.4 \%$ in the placebo group, $P<0.001){ }^{20}$ All patients received aspirin $75 \mathrm{mg}-325 \mathrm{mg}$ per day. A significantly higher incidence of major bleeding was associated with clopidogrel (3.7\%) compared with placebo $(2.7 \%, P=0.001)$. These findings form part of the basis for the practices recommended in the ACC/AHA guidelines of withholding clopidogrel for at least 5 days before $C A B G$ surgery and continuing the drug for 12 months in patients with NSTEMI or UA. 2,18

The GP IIb/IIla receptor is ubiquitous on the platelet surface. Fibrinogen binds to the receptor on multiple activated platelets, causing platelet aggregation regardless of what pathway led to platelet activation (i.e., the GP IIb/IIla receptor is the final common pathway for platelet aggregation). The receptor is blocked by GP IIb/IIIa inhibitors, preventing platelet aggregation. 
Three GP IIb/IIIa inhibitors (abciximab, eptifibatide, tirofiban) are available in the United States. Platelet inhibition from GP IIb/ IIIa inhibitors begins promptly after initiation of drug therapy. Stopping eptifibatide and tirofiban, but not abciximab, infusion returns platelet function to normal within several hours after discontinuation of therapy. ${ }^{21,22}$

GP IIb/IIla inhibitors have been evaluated primarily in patients with NSTEMI or UA who undergo PCI. Support for the use of GP IIb/IIIa inhibitors in patients with NSTEMI or UA who are initially treated conservatively with medical therapy is agent specific. Abciximab should not be used in patients with NSTEMI or UA if a conservative medical strategy is planned. The drug is approved by the FDA only as an adjunct to PCI. ${ }^{21}$ Patients with NSTEMI or UA who have high-risk features, worsening symptoms, or in whom coronary angiography is considered but will be delayed should receive eptifibatide or tirofiban instead of abciximab. ${ }^{18}$

Eptifibatide and tirofiban are approved by the FDA for use in combination with aspirin and UFH for the management of patients with NSTEMI or UA who are treated medically or invasively. 22,23

\section{Anticoagulant Therapies}

Every patient presenting with NSTEMI or UA should be treated with an anticoagulant in addition to antiplatelet therapy. The choice of anticoagulant often depends on whether and when cardiac catheterization is planned.

The anticoagulant with the longest history of use in patients with NSTEMI or UA is UFH. In the Antithrombotic Therapy in Acute Coronary Syndromes study of 214 patients with NSTEMI or UA, UFH plus aspirin reduced the 14-day incidence of recurrent angina with ECG changes, MI, or death from $27 \%$ with aspirin alone to $10.5 \%$ with combination therapy $(P=0.004) .{ }^{24}$

UFH is administered as a weight-based intravenous bolus dose followed by maintenance intravenous infusion, with a goal aPTT between 50 and 70 seconds. ${ }^{18}$ The advantage of UFH lies in the ease of regulating its anticoagulant effect during cardiac catheterization. The level of anticoagulation can be easily monitored with a rapid point-of-care assay, the activated clotting time (ACT). There is considerable experience with the use of UFH in the cardiac catheterization laboratory. Its anticoagulant effects can be readily reversed with protamine if bleeding complications arise.

The use of enoxaparin in patients who proceed to the cardiac catheterization laboratory is more problematic than UFH. There is no reliable, widely available point-of-care testing to monitor the anticoagulant effects of enoxaparin, and these effects are much less reversible with protamine than are the effects of UFH. ${ }^{25}$

The use of enoxaparin and UFH was compared in the SYNERGY trial, a randomized, open-label study of more than 10,000 patients with NSTEMI who planned to undergo an early invasive strategy. ${ }^{26}$ Patients were randomly assigned to receive enoxaparin $1 \mathrm{mg}$ per $\mathrm{kg}$ subcutaneously every 12 hours or UFH 60 units per $\mathrm{kg}$ (not to exceed 5,000 units) intravenously followed by 12 units per kg per hour (not to exceed 1,000 units per hour) intravenously. Platelet GP IIb/IIIa inhibitors also were used during coronary interventions. There was no significant difference between enoxaparin and UFH in the 30-day incidence of death or nonfatal MI (14.0\% and 14.5\%, respectively; $P=0.400)$. A significantly higher incidence of TIMI major bleeding at 30 days was associated with enoxaparin (9.1\%) compared with UFH $(7.6 \%, P=0.008)$. In patients who received the same therapy consistently throughout the SYNERGY study (i.e., no crossover to heparin or vice versa), enoxaparin significantly reduced the 30-day incidence of death or nonfatal MI by 18\% from $15.9 \%$ in the UFH group to $13.3 \%$ in the enoxaparin group. Seventeen percent of patients in both treatment groups required transfusions. Therefore, some say it is prudent to adhere to a consistent anticoagulation strategy before and during cardiac catheterization in patients with NSTEMI or UA (i.e., use UFH in patients scheduled to undergo cardiac catheterization). On the other hand, many interventionalists are much more comfortable using UFH, and any use of other therapies risks a crossover and could affect outcomes in a negative way, such as the outcomes of those patients who crossed over in this study.

The role of fondaparinux in patients with NSTEMI was investigated in the OASIS-5 trial, a randomized, double-blind, noninferiority study of $>20,000$ patients who received fondaparinux $2.5 \mathrm{mg}$ subcutaneously daily or enoxaparin $1 \mathrm{mg}$ subcutaneously twice daily for a mean of 6 days. ${ }^{27}$ Approximately $72 \%$ of patients underwent coronary angiography, $40 \%$ underwent $\mathrm{PCI}$, and $15 \%$ underwent CABG surgery during or after hospitalization. Fondaparinux was judged to be noninferior to enoxaparin based on a similar rate of death, MI, or refractory ischemia after 9 days (5.8\% with fondaparinux vs. 5.7\% with enoxaparin). Fondaparinux was associated with significantly lower rates of major bleeding after 9 days $(2.2 \%$ vs. $4.1 \%$ with enoxaparin, $P<0.001)$ and death after 30 days $(P=0.02)$ and 180 days $(P=0.05)$ compared with enoxaparin. The practice of switching to UFH during coronary intervention in the enoxaparin group may partly explain the increased incidence of bleeding. Patients in the fondaparinux group were eligible to receive additional fondaparinux during the coronary intervention, but they did not receive UFH.

In OASIS-5 patients who underwent PCI, the incidence of catheter-related thrombi was significantly higher with the use of fondaparinux $(0.9 \%)$ than with enoxaparin $(0.4 \%, P=0.001) .{ }^{27}$ This finding limits the use of fondaparinux in patients with NSTEMI because most of these patients in the United States undergo cardiac catheterization. Fondaparinux or enoxaparin is preferred over UFH for patients with NSTEMI or UA in whom cardiac catheterization is not planned. ${ }^{18}$ In the ACC/ AHA guidelines for the management of UA or NSTEMI, the use of fondaparinux is preferred in patients at increased risk for 
bleeding when a conservative approach is chosen. ${ }^{18}$ The use of fondaparinux in patients with NSTEMI or UA is not approved by the FDA, but it is endorsed by the ACC/AHA. ${ }^{16,18}$

\section{Therapies Specific to PCI}

The use of PCI as the primary reperfusion strategy in patients with STEMI is associated with a significantly lower short-term mortality rate $(5 \%)$ than the use of thrombolytic agents for reperfusion $(7 \%, P<0.001) .{ }^{28} \mathrm{PCI}$ carries a significantly lower risk of nonfatal reinfarction (3\% vs. $7 \%$ with thrombolytic agents, $P<0.001)$ and stroke ( $1 \%$ vs. $2 \%$ with thrombolytic agents, $P<0.001)$.

Early invasive strategies have been compared with more conservative strategies in patients with NSTEMI or UA. In the TACTICS-TIMI 18 study of 2,220 patients with NSTEMI or UA, the rate of death, nonfatal MI, or rehospitalization for ACS at 6 months (the primary endpoint) was significantly lower with use of an early invasive strategy with cardiac catheterization within 4-48 hours and revascularization if needed (15.9\%) than with use of a more conservative strategy of catheterization only for patients with evidence of recurrent ischemia or abnormal stress test results (19.4\%, $P=0.025){ }^{29}$ Troponin T levels were elevated in $54 \%$ of patients (i.e., $54 \%$ of enrollees had NSTEMI), and the benefit of the early invasive strategy was observed only in these patients. In patients without elevated troponin T levels (i.e., UA patients), there was no significant difference between the 2 interventions in the primary endpoint $(P=0.46)$.

Stent implantation after revascularization of a coronary artery covers the ruptured plaque and prevents it from coming in contact with constituents in the bloodstream that promote thrombosis. This approach obviates the need for prolonged anticoagulation. Nevertheless, the stent itself can act as a nidus for thrombosis; therefore, antiplatelet therapy is required to maintain patency of the reopened vessel.

Patients who undergo coronary intervention and stent implantation present a unique set of therapeutic challenges because of the need to balance the prevention of thrombosis with minimization of the risk of bleeding. A tremendous risk for clot formation exists when catheters, coronary wires, balloons, and stents are introduced into small coronary arteries. Introducing these devices into the femoral artery in the leg carries a potential for arterial injury and bleeding complications. Anticoagulants used during coronary interventions ideally have a rapid onset and a short half-life, can be easily monitored, and are associated with a low risk of bleeding.

\section{Antiplatelet Therapies}

Patients undergoing coronary stent deployment should receive aspirin $162 \mathrm{mg}-325 \mathrm{mg}$ preferably as a chewable, nonenteric formulation prior to the procedure..$^{18}$ Aspirin $75 \mathrm{mg}-325 \mathrm{mg}$ per day should be continued indefinitely after stent insertion.

Platelet GP IIb/IIla inhibitors are routinely used during PCI in patients with STEMI and NSTEMI. ${ }^{2,18}$ Their efficacy in reducing the rate of death, MI, and the need for repeat revascularization is well established. ${ }^{30}$ These agents increase the risk of thrombocytopenia and minor bleeding, but they do not increase the risk of major bleeding. ${ }^{30}$

The need for a GP IIb/IIIa inhibitor in patients with NSTEMI or UA who receive loading doses of clopidogrel and aspirin before coronary intervention has been questioned. In the randomized, double-blind ISAR-REACT-2 trial of 2,022 patients with NSTEMI or UA, a 600-mg loading dose of clopidogrel was administered at least 2 hours prior to $\mathrm{PCI} .{ }^{31}$ Patients were randomly assigned to receive abciximab plus UFH or placebo plus UFH. All patients received aspirin. After 30 days, the incidence of death, MI, or urgent target vessel revascularization (the primary endpoint) was $8.9 \%$ in the abciximab group and $11.9 \%$ in the placebo group $(P=0.03)$, representing a $25 \%$ reduction in risk of the primary endpoint with the use of abciximab. The benefit of abciximab was present even 12 months later. ${ }^{32}$ This difference was observed only in patients with elevated troponin levels (i.e., patients with NSTEMI instead of patients with UA). No significant differences were found between the 2 treatment groups in the risk of major bleeding, risk of minor bleeding, or need for transfusion. These findings provide support for the use of GP IIb/IIla inhibitors in addition to aspirin and clopidogrel in patients with NSTEMI and elevated troponin levels or other high-risk features. ${ }^{18}$

\section{Anticoagulant Therapies}

The most commonly used anticoagulant during any coronary intervention remains UFH because it is easy to administer, has a short half-life, and its anticoagulant effects can be rapidly assessed using the ACT assay and readily reversed using protamine. In the cardiac catheterization laboratory, the track record for UFH is well established, and clinicians are familiar and comfortable with the agent.

Enoxaparin has not gained popularity as an anticoagulant agent for routine use during PCI because its anticoagulant effect cannot be readily monitored. Its half-life is relatively long, causing concern about the risk of bleeding during removal of devices from arteries after coronary intervention. Nevertheless, some patients with NSTEMI or UA who are initially treated with enoxaparin eventually undergo coronary intervention. The current ACC/AHA guidelines stipulate that if 8-12 hours have elapsed since the last subcutaneous dose of enoxaparin, an intravenous bolus dose of $0.3 \mathrm{mg}$ per $\mathrm{kg}$ should be administered. 2,18,33

Experience from the OASIS-5 trial raised concerns about catheter-related thrombi in patients with NSTEMI or UA who received fondaparinux and underwent PCI. ${ }^{27}$ Until more safety data become available, fondaparinux is unlikely to be widely used in the cardiac catheterization laboratory.

Another agent that has recently been evaluated is bivalirudin, a synthetic, reversible direct thrombin inhibitor that binds to circulating and clot-bound thrombin, preventing further steps in the coagulation process. ${ }^{34}$ It has an immediate onset of action 
after intravenous administration and a short half-life ( 25 minutes in patients with normal renal function). ${ }^{35}$

Bivalirudin alone and in combination with a GP IIb/IIla inhibitor were compared with heparin (UFH or enoxaparin) plus a GP IIb/IIIa inhibitor in the ACUITY study, a randomized, controlled, noninferiority study of more than 13,000 patients with NSTEMI scheduled to undergo angiography. ${ }^{36}$ Most patients received clopidogrel. The 30-day incidence of death from any cause, MI, or unplanned revascularization for ischemia (i.e., ischemic outcomes) was $7.8 \%$ with bivalirudin alone, $7.7 \%$ with bivalirudin plus a GP IIb/IIla inhibitor, and $7.3 \%$ with UFH or enoxaparin plus a GP IIb/IIIa inhibitor. The 30-day incidence of major bleeding with bivalirudin alone was significantly lower at $3 \%(P<0.001)$ compared with a $5.3 \%$ bleeding rate in bivalirudin plus a GP IIb/IIIa inhibitor and a rate of 5.7\% when using UFH or enoxaparin plus a GP IIb/IIIa inhibitor. The use of a liberal definition of major bleeding may have influenced these results. Nevertheless, bivalirudin alone may be chosen over bivalirudin plus a GP IIb/IIIa inhibitor and UFH or enoxaparin plus a GP IIb/IIIa inhibitor in patients with NSTEMI who undergo early invasive procedures because it is at least as effective for reducing ischemic outcomes with a lower risk for major bleeding.

About one half (56\%) of ACUITY enrollees underwent PCI, and roughly two thirds of these patients received clopidogrel before PCI. ${ }^{37}$ In patients with clopidogrel exposure before PCI, the 30-day incidence of ischemic outcomes was similar to bivalirudin alone (8.1\%) and UFH or enoxaparin plus a GP IIb/ IIla inhibitor (8.4\%). The 30-day rate of major bleeding was 3.6\% and $7.2 \%$, respectively. However, in patients without clopidogrel exposure before $\mathrm{PCI}$, the incidence of ischemic outcomes was 9.6\% with bivalirudin alone and $7.4 \%$ with UFH or enoxaparin plus a GP IIb/IIIa inhibitor. The 30-day rate of major bleeding in patients without clopidogrel exposure before PCI was 3.7\% with bivalirudin alone and $5.5 \%$ with UFH or enoxaparin plus a GP IIb/IIIa inhibitor. These findings suggest that clopidogrel or a GP IIb/IIIa inhibitor is needed in patients with NSTEMI who undergo PCI; bivalirudin alone is inadequate.

\section{Long-Term Antiplatelet Therapy After Coronary Stenting}

A stent implanted in a coronary artery represents a foreign body in the prothrombotic milieu of the bloodstream of a patient recovering from ACS. In the early era of coronary stenting, the most feared complication was stent thrombosis, resulting in occlusion of the stent and potentially fatal MI.

Coronary stents are made of stainless steel or a cobaltchromium alloy and are known as bare-metal stents. Dual antiplatelet therapy was provided for 30 days. After that time, a layer of endothelial cells of the native coronary artery had formed on the stent, preventing the stent from coming in contact with prothrombotic constituents in the bloodstream. The risk of stent thrombosis after 30 days decreased sufficiently to warrant therapy with aspirin alone.
Bare-metal stents were associated with an increased risk of restenosis due to the proliferation of smooth muscle cells in the arterial wall in response to injury caused by stent implantation. Drug-eluting stents were developed to minimize smooth muscle cell proliferation and prevent stent restenosis. These stents are coated with a polymer-eluting drug that inhibits cell proliferation. Drug-eluting stents have had a tremendous impact on the long-term efficacy of coronary interventions, and they have revolutionized interventional cardiology's management of patients with ACS, allowing the treatment of complex coronary artery blockages. The introduction of sirolimus- and paclitaxel-coated stents in 2003 and 2004, respectively, significantly reduced the risk of stent restenosis by up to $75 \%$ compared with approximately $10 \%$ for bare-metal stents. ${ }^{38,39}$ Members of a newer generation of drug-eluting stents (e.g., a zotarolimus-coated stent) possess anti-inflammatory, antimigratory, antiproliferative, or prohealing effects. ${ }^{40,41}$ However, one disadvantage is that the protective endothelial cell growth over the stent is also reduced, requiring longer dual antiplatelet therapy.

Risk of stent thrombosis is less than $1 \%$ within the first year after bare-metal stent implantation. ${ }^{42}$ In an analysis of safety data by the FDA, a small increase in stent thrombosis was found after 1 year in patients with drug-eluting stents compared with patients with bare-metal stents, but the increase was not associated with an increased risk for death or MI. ${ }^{43}$

The ACC/AHA guidelines recommend aspirin $162 \mathrm{mg}-325 \mathrm{mg}$ per day for 1 month after bare-metal stent implantation, 3 months after sirolimus-eluting stent implantation, and 6 months after paclitaxel-eluting stent implantation, followed by $75 \mathrm{mg}-162 \mathrm{mg}$ per day indefinitely in patients with all types of stents.,18 After reviewing the safety of drug-eluting stents, an FDA panel recommended a 12-month course of dual antiplatelet therapy after drug-eluting stent implantation. In patients in whom there is concern about an increased risk for bleeding, a lower aspirin dosage (75 mg-162 mg per day) may be used during the initial period after stent implantation. ${ }^{2}$ Clopidogrel $75 \mathrm{mg}$ per day is recommended for at least 12 months after drug-eluting stent implantation unless the patient is at high risk for bleeding. ${ }^{2,18}$

The risk of stent thrombosis after premature discontinuation of antiplatelet therapy is high and potentially catastrophic. ${ }^{44}$ Discontinuation of clopidogrel, aspirin, or both due to adverse effects or plans to undergo surgery or invasive procedures (e.g., colonoscopy, biopsy) should be attempted only after consultation with a cardiologist.

\section{Conclusions}

The increasing complexity of the duration of treatment, dosages, and combinations of pharmacological agents available for management of ACS poses a challenge to clinicians when selecting appropriate antiplatelet and anticoagulant therapy. Although currently available, evidence-based guidelines are extremely helpful in directing treatment decisions, antiplatelet and anticoagulant 
therapies must be selected based on an assessment of the risk for recurrent coronary events, death, and bleeding in a specific individual.

\section{REFERENCES}

1. Rosamond W, Flegal K, Furie K, et al. Heart disease and stroke statistics_-2008 update: a report from the American Heart Association Statistics Committee and Stroke Statistics Subcommittee. Circulation. 2008;117(4):e25-146.

2. Antman EM, Hand M, Armstrong PW, et al. 2007 Focused update of the ACC/AHA 2004 guidelines for the management of patients with ST-elevation myocardial infarction: a report of the American College of Cardiology/ American Heart Association Task Force on Practice Guidelines. Circulation. 2008;117:296-329.

3. Fibrinolytic Therapy Trialists' (FTT) Collaborative Group. Indications for fibrinolytic therapy in suspected acute myocardial infarction: collaborative overview of early mortality and major morbidity results from all randomised trials of more than 1000 patients. Lancet. 1994;343:311-22.

4. McEvoy GK, ed. Aspirin. In: AHFS Drug Information 2008. Bethesda, MD: American Society of Health-System Pharmacists; 2008:2073-89.

5. ISIS-2 (Second International Study of Infarct Survival) Collaborative Group. Randomised trial of intravenous streptokinase, oral aspirin, both, or neither among 17,187 cases of suspected acute myocardial infarction: ISIS-2. Lancet. 1988;2:349-60.

6. McEvoy GK, ed. Clopidogrel bisulfate. In: AHFS Drug Information 2008. Bethesda, MD: American Society of Health-System Pharmacists; 2008:1520-24.

7. McEvoy GK, ed. Ticlopidine hydrochloride. In: AHFS Drug Information 2008. Bethesda, MD: American Society of Health-System Pharmacists; 2008:1531-34.

8. Patti G, Colonna G, Pasceri V, et al. Randomized trial of high loading dose of clopidogrel for reduction of periprocedural myocardial infarction in patients undergoing coronary intervention: results from the ARMYDA-2 (Antiplatelet therapy for Reduction of Myocardial Damage during Angioplasty) study. Circulation. 2005;111:2099-106.

9. Hochholzer W, Trenk D, Frundi D, et al. Time dependence of platelet inhibition after a 600-mg loading dose of clopidogrel in a large, unselected cohort of candidates for percutaneous coronary intervention. Circulation. 2005;111:2560-64

10. Chen ZM, Jiang LX, Chen YP, et al. Addition of clopidogrel to aspirin in 45,852 patients with acute myocardial infarction: randomised placebocontrolled trial. Lancet. 2005;366:1607-21.

11. Sabatine MS, Cannon CP, Gibson CM, et al. Addition of clopidogrel to aspirin and fibrinolytic therapy for myocardial infarction with ST-segment elevation. N Engl J Med. 2005;352:1179-89.

12. Nutescu E, Dager W. Heparin, low molecular weight heparin, and fondaparinux. In: Gulseth M, ed. Managing Anticoagulation Patients in the Hospital. Bethesda, MD: American Society of Health-System Pharmacists; 2007:177-202.

13. Assessment of the Safety and Efficacy of a New Thrombolytic Regimen (ASSENT)-3 Investigators. Efficacy and safety of tenecteplase in combination with enoxaparin, abciximab, or unfractionated heparin: the ASSENT-3 randomised trial in acute myocardial infarction. Lancet. 2001;358:605-13.

14. Antman EM, Morrow DA, McCabe CH, et al. Enoxaparin versus unfractionated heparin with fibrinolysis for ST-elevation myocardial infarction. N Engl J Med. 2006;354:1477-88.

15. White HD, Braunwald E, Murphy SA, et al. Enoxaparin vs. unfractionated heparin with fibrinolysis for ST-elevation myocardial infarction in elderly and younger patients: results from ExTRACT-TIMI 25. Eur Heart J. 2007;28:1066-71.
16. GlaxoSmithKline. Arixtra [package insert]. Research Triangle Park, NC: October 2005.

17. Yusuf S, Mehta SR, Chrolavicius S, et al. Effects of fondaparinux on mortality and reinfarction in patients with acute ST-segment elevation myocardial infarction: the OASIS-6 randomized trial. JAMA. 2006;295:1519-30.

18. Anderson JL, Adams CD, Antman EM, et al. ACC/AHA 2007 guidelines for the management of patients with unstable angina/non-ST-elevation myocardial infarction: a report of the American College of Cardiology/ American Heart Association Task Force on Practice Guidelines. Circulation. 2007;116:e148-304

19. Antman EM, Cohen M, Bernink PJ, et al. The TIMI risk score for unstable angina/non-ST-elevation MI: a method for prognostication and therapeutic decision making. JAMA. 2000;284:835-42.

20. Yusuf S, Zhao F, Mehta SR, et al. Effects of clopidogrel in addition to aspirin in patients with acute coronary syndromes without ST-segment elevation. N Engl J Med. 2001;345:494-502.

21. Eli Lilly and Company. ReoPro [package insert]. Indianapolis, IN: November 2005.

22. Merck \& Co., Inc. Aggrastat [package insert]. Whitehouse Station, NJ: May 2003

23. Schering Corporation. Intregrilin [package insert]. Kenilworth, NJ: June 2006.

24. Cohen M, Adams PC, Parry G, et al. Combination antithrombotic therapy in unstable rest angina and non-Q-wave infarction in nonprior aspirin users. Primary end points analysis from the ATACS trial. Antithrombotic Therapy in Acute Coronary Syndromes Research Group. Circulation. 1994;89:81-88.

25. McEvoy GK, ed. Protamine sulfate. In: AHFS Drug Information 2008. Bethesda, MD: American Society of Health-System Pharmacists; 2008:1595-97.

26. Ferguson JJ, Califf RM, Antman EM, et al. Enoxaparin vs. unfractionated heparin in high-risk patients with non-ST-segment elevation acute coronary syndromes managed with an intended early invasive strategy: primary results of the SYNERGY randomized trial. JAMA. 2004;292:45-54.

27. Yusuf S, Mehta SR, Chrolavicius S, et al., for the Fifth Organization to Assess Strategies in Acute Ischemic Syndromes Investigators. Comparison of fondaparinux and enoxaparin in acute coronary syndromes. N Engl J Med. 2006;354:1464-76.

28. Keeley EC, Boura JA, Grines CL. Primary angioplasty versus intravenous thrombolytic therapy for acute myocardial infarction: a quantitative review of 23 randomised trials. Lancet. 2003;361:13-20.

29. Cannon CP, Weintraub WS, Demopoulos LA, et al. Comparison of early invasive and conservative strategies in patients with unstable coronary syndromes treated with the glycoprotein IIb/IIIa inhibitor tirofiban. N Engl J Med. 2001;344:1879-87.

30. Labinaz M, Ho C, Banerjee S, et al. Meta-analysis of clinical efficacy and bleeding risk with intravenous glycoprotein IIb/IIIa antagonists for percutaneous coronary intervention. Can J Cardiol. 2007;23:963-70.

31. Kastrati A, Mehilli J, Neumann FJ, et al. Abciximab in patients with acute coronary syndromes undergoing percutaneous coronary intervention after clopidogrel pretreatment: the ISAR-REACT 2 randomized trial. JAMA. 2006;295:1531-38.

32. Ndrepepa G, Kastrati A, Mehilli J, et al. One-year clinical outcomes with abciximab vs. placebo in patients with non-ST-segment elevation acute coronary syndromes undergoing percutaneous coronary intervention after pretreatment with clopidogrel: results of the ISAR-REACT 2 randomized trial. Eur Heart J. 2008;29(4):455-61

33. Martin JL, Fry ET, Sanderink GJ, et al. Reliable anticoagulation with enoxaparin in patients undergoing percutaneous coronary intervention: the pharmacokinetics of enoxaparin in PCI (PEPCI) study. Catheter Cardiovasc Interv. 2004;61:163-70. 
34. Dager W. Essential direct thrombin inhibitor knowledge. In: Gulseth M, ed. Managing Anticoagulation Patients in the Hospital. Bethesda, MD: American Society of Health-System Pharmacists; 2007:203-13.

35. The Medicines Company. Angiomax [package insert]. Parsippany, NJ: December 2005

36. Stone GW, McLaurin BT, Cox DA, et al. Bivalirudin for patients with acute coronary syndromes. N Engl J Med. 2006;355:2203-16.

37. Stone GW, White HD, Ohman EM, et al. Bivalirudin in patients with acute coronary syndromes undergoing percutaneous coronary intervention: a subgroup analysis from the Acute Catheterization and Urgent Intervention Triage strategy (ACUITY) trial. Lancet. 2007;369:907-19.

38. Roiron C, Sanchez P, Bouzamondo A, et al. Drug eluting stents: an updated meta-analysis of randomised controlled trials. Heart. 2006;92:641-49.

39. Dibra A, Kastrati A, Alfonso F, et al. Effectiveness of drug-eluting stents in patients with bare-metal in-stent restenosis: meta-analysis of randomized trials. J Am Coll Cardiol. 2007;49:616-23.
40. Chen YW, Smith ML, Sheets M, et al. Zotarolimus, a novel sirolimus analogue with potent anti-proliferative activity on coronary smooth muscle cells and reduced potential for systemic immunosuppression. J Cardiovasc Pharmacol. 2007:49:228-35.

41. Anis RR, Karsch KR, Oberhoff M. An update on clinical and pharmacological aspects of drug-eluting stents. Cardiovasc Hematol Disord Drug Targets. 2006;6:245-55.

42. Doyle B, Rihal CS, O'Sullivan CJ, et al. Outcomes of stent thrombosis and restenosis during extended follow-up of patients treated with bare-metal coronary stents. Circulation. 2007;116:2391-98.

43. U.S. Food and Drug Administration. Update to FDA statement on coronary drug-eluting stents. Available at: www.fda.gov/cdrh/news/010407.html. Accessed March 16, 2008.

44. Spertus JA, Kettelkamp R, Vance C, et al. Prevalence, predictors, and outcomes of premature discontinuation of thienopyridine therapy after drug-eluting stent placement: results from the PREMIER registry. Circulation. 2006;113:2803-09. 